Discrete Comput Geom 29:161-173 (2003)

DOI: $10.1007 /$ s00454-002-2828-y

\title{
A Constant Bound for Geometric Permutations of Disjoint Unit Balls
}

\author{
Meir Katchalski, ${ }^{1}$ Subhash Suri, ${ }^{2}$ and Yunhong Zhou $^{3}$ \\ ${ }^{1}$ Faculty of Mathematics, Technion, \\ Haifa 32000, Israel \\ meirk@ techunix.technion.ac.il \\ ${ }^{2}$ Computer Science Department, University of California, \\ Santa Barbara, CA 93106, USA \\ suri@cs.ucsb.edu \\ ${ }^{3}$ Compaq Systems Research Center, \\ 130 Lytton Ave, Palo Alto, CA 94301, USA \\ yzhou@pa.dec.com
}

\begin{abstract}
We prove that a set of $n$ disjoint unit balls in $\mathcal{R}^{d}$ admits at most four distinct geometric permutations, or line transversals, thus settling a long-standing conjecture in combinatorial geometry. The constant bound significantly improves upon the $\Theta\left(n^{d-1}\right)$ bound for disjoint balls of unrestricted radii.
\end{abstract}

\section{Introduction}

An oriented line $\ell$ is called a line transversal for a set $\mathcal{S}$ of bodies in $\mathcal{R}^{d}$ if it intersects every member of $\mathcal{S}$. If the objects in $\mathcal{S}$ are pairwise disjoint convex bodies, then a line transversal of $\mathcal{S}$ induces two linear orderings, which are the orders in which $\ell$ meets the members of $\mathcal{S}$. The two orders induced by a line transversal are essentially the same (one is the reverse of the other), and so they are together called a single geometric permutation.

Let $g_{d}(n)$ denote the maximum number of geometric permutations, where the maximum is taken over all such families $\mathcal{S}$ of size $n$ in $\mathcal{R}^{d}$. For the planar case $d=2$, we know the exact bound $g_{2}(n)=2 n-2$ [9], [4]. For $d>2$, the best upper bound known for general convex bodies is $O\left(n^{2 d-2}\right)$ by Wenger [13]. Katchalski et al. [8] showed that $n$ disjoint convex bodies in $\mathcal{R}^{d}$ can realize $\Omega\left(n^{d-1}\right)$ geometric permutations. Thus, there remains a substantial gap between the known upper bound $O\left(n^{2 d-2}\right)$ and lower bound $\Omega\left(n^{d-1}\right)$ on geometric permutations.

This gap motivated Smorodinsky et al. [11] to consider a natural, but specialized, family of convex bodies: balls. They constructed a family of disjoint balls accepting $\Omega\left(n^{d-1}\right)$ 
geometric permutations. Furthermore, they were able to prove a matching asymptotic upper bound, thus showing that the maximum number of geometric permutations for collections of $n$ disjoint balls in $\mathcal{R}^{d}$ is $\Theta\left(n^{d-1}\right)$. Recently Katz and Varadarajan [10] generalized the same result to $\alpha$-fat convex bodies in $\mathcal{R}^{d}$.

An interesting special case of the problem is when all balls in $\mathcal{S}$ are congruent (say, unit balls). Smorodinsky et al. [11] show that the maximum number of geometric permutations for $n$ unit disks in the plane is only 2, for sufficiently large $n$. (An independent proof of this fact is also given by Katchalski and Asinowski [2].) It has been conjectured that unit balls in any dimension admit only a constant number of permutations, but this conjecture has been open for dimensions $d \geq 3$.

In this paper we settle this long-standing open conjecture by proving that the maximum number of geometric permutations for a family of $n$ pairwise disjoint unit radius balls in $\mathcal{R}^{d}$ is four, for $n$ a sufficiently large constant that depends on the dimension $d$. The number of permutations, however, is independent of $d$. Thus, the number of geometric permutations drops precipitously from $\Theta\left(n^{d-1}\right)$ to a small constant if all the balls are congruent.

While in combinatorial geometry line transversals and geometric permutations have been studied primarily for their mathematical appeal, they also find practical applications in computing visibility information in three-dimensional computer games and architectural walkthroughs. These applications decompose the space into boxes, and then try to compute visibility between various boxes. The openings through which light can pass, such as windows, doors and the tops of stairwells in a building model, are modeled by axis-aligned rectangular portals on the faces of these boxes; all other objects are considered opaque. The visibility among boxes is computed from the visibility between these portals. See [12] for details. Similar ideas are used in various video games as well.

The portal visibility approach motivated research on the problem of determining whether a given set of objects can be stabbed by a line; that is, does the set have a line transversal. The line transversal problem has also been studied as a generalization of the classical Helly Theorem of combinatorial geometry [3]. For a survey of geometric transversal theory, see [5] and [14]. Amenta [1] and Hohmeyer and Teller [6] have given efficient algorithms for computing line transversals.

Our paper is organized as follows. In Section 2 we establish a series of properites for line transversals, prove a key technical lemma, and introduce the concept of switched pairs. In Section 3 we prove our major result: an upper bound on the number of geometric permutations for congruent balls. We conclude in Section 4.

In June 2000 we circulated an earlier draft of our paper, which established an upper bound of 16 on the number of unit ball permutations. Soon after that, Huang et al. improved the bound to four permutations by a simple tightening of our Lemma 2.5. After learning of their result, we also independently achieved the same improvement, as presented in this paper. Our work was presented at SODA 2001 [15], as was the paper by Huang et al. [7].

\section{Properties of Line Transversals}

In this section we establish a series of properties for line transversals of a family of disjoint unit balls in $\mathcal{R}^{d}$, for $d \geq 2$. The key observation here is that if $n$, the number of unit balls, is large enough, then all balls will line up very closely to a single line. 
Lemma 2.1. Let $\mathcal{S}$ be a family of $n$ disjoint unit balls in $\mathcal{R}^{d}$ with at least one geometric permutation. Then the distance between the centers of a farthest pair of balls is $\Omega(n)$, where the constant depends on $d$.

Proof. Let $o_{1}, o_{2}$ denote the centers of the two balls that form the farthest pair in $\mathcal{S}$, let $D=\operatorname{dist}\left(o_{1}, o_{2}\right)$, and let $\ell$ be a line transversal of $\mathcal{S}$. The projection of $\mathcal{S}$ onto $\ell$ has length at most $2+D$, and thus the set $\mathcal{S}$ is contained within a radius 2 cylinder of height $2+D$. If $V_{d}$ denotes the volume of a unit ball in $\mathcal{R}^{d}$, then the volume of this cylinder is $V_{d-1}(2+D) 2^{d-1}$. Since $n$ unit balls are contained in this cylinder, and $d$ is a constant we get $V_{d-1}(2+D) 2^{d-1} \geq n V_{d}$, which implies $D \geq\left(V_{d} / 2^{d-1} V_{d-1}\right) n-2=\Omega(n)$. This completes the proof.

Lemma 2.2. Let $B_{1}, B_{2}$ be disjoint unit balls in $\mathcal{R}^{d}$ with centers $o_{1}$ and $o_{2}$, respectively, and let $\ell$ be a line that intersects both of them. Let $L=\operatorname{dist}\left(o_{1}, o_{2}\right)$, and let $\theta$ denote the angle between $\ell$ and the line containing the centers $o_{1}, o_{2}$. Then we have $\sin \theta \leq 2 / L$, and $\theta$ achieves its maximum value when $\ell$ is a common tangent of $B_{1}, B_{2}$ through the midpoint of segment $o_{1} o_{2}$.

Proof. This is a well-known result. For completeness, we give an outline proof here.

First we reduce the problem from general dimension $\mathcal{R}^{d}$ to $\mathcal{R}^{2}$. Let $\ell_{o}$ be the line connecting centers $o_{1}, o_{2}$. Consider the two-dimensional plane $\pi$ passing through $\ell$ and parallel to $\ell_{0}$. The intersections between plane $\pi$ and balls $B_{1}, B_{2}$ are congruent disks $D_{1}, D_{2}$ centered at $o_{1}^{\prime}, o_{2}^{\prime}$. Let $\ell_{o}^{\prime}$ be the line containing $o_{1}^{\prime}, o_{2}^{\prime}$. It is easy to see that $\ell_{o}$ is parallel to $\ell_{o}^{\prime}$ and $\ell_{o}^{\prime} \subset \pi$. Thus the angle between $\ell$ and $\ell_{o}$ equals the angle between $\ell$ and $\ell_{o}^{\prime}$. $\ell$ intersects both $D_{1}$ and $D_{2}$. Now we only need to focus on the two-dimensional plane $\pi$, thus reducing the problem from $\mathcal{R}^{d}$ to $\mathcal{R}^{2}$.

The maximum-angle line transversal for any two disjoint congruent disks in $\mathcal{R}^{2}$ must be an inner tangent of these disks. If it is not true, then we can first translate the line such that it passes throught the midpoint $o^{\prime}$ of segment $o_{1}^{\prime} o_{2}^{\prime}$. The translated line intersects at least one disk as it moves closer to one disk. Because the translated line is in a symmetric position with respect to these disks, it intersects both disks. After the translation we rotate the line around $o^{\prime}$ such that it becomes tangent to both disks. The translation preserves the angle between the line transversal with $\ell_{o}^{\prime}$ and the rotation increases its angle with $\ell_{o}^{\prime}$. Thus the maximum angle is achieved at the position of one inner tangent.

Equality is achieved only when the sizes of $D_{1}$ and $D_{2}$ are maximized and $\ell$ is an inner tangent of $D_{1}$ and $D_{2}$. In this case $\ell$ is coplanar with $o_{1} o_{2}$. Furthermore, $\ell$ is a common tangent of $B_{1}$ and $B_{2}$ passing through the midpoint of $o_{1} o_{2}$. It is easy to verify that the maximum angle $\theta$ satisfies $\sin \theta=2 / L$.

Lemma 2.3. Let $\mathcal{S}$ be a set of $n$ pairwise disjoint unit balls in $\mathcal{R}^{d}$ with at least one geometric permutation, and assume that the $z$-axis is the line connecting the centers of the farthest pair of balls in $\mathcal{S}$. Then the angle between any line transversal of $\mathcal{S}$ and the $z$-axis is bounded by $O(1 / n)$; consequently, the angle between any two line transversals of $\mathcal{S}$ is also $O(1 / n)$. 
Proof. Let $o_{1}, o_{2}$ denote the centers of the two balls that form the farthest pair, and let $D=\operatorname{dist}\left(o_{1}, o_{2}\right)$. By Lemma 2.1, we have that $D=\Omega(n)$. Given an arbitrary line transversal $\ell$, if $\theta$ is the angle between $\ell$ and the $z$-axis, then by Lemma 2.2 we have $\sin \theta \leq 2 / D$. Thus,

$$
\theta \leq \frac{\pi}{2} \sin \theta \leq \frac{\pi}{D}=O(1 / n)
$$

The angle between any two line transversals of $\mathcal{S}$ is at most twice the maximal angle between one line transversal and the $z$-axis, and so the lemma follows.

\subsection{One Technical Lemma}

Given two disjoint unit balls in $\mathcal{R}^{d}$, the center of gravity of these balls is the midpoint of the segment joining the two ball centers, and lies outside of both balls. In order to bound geometric permutations quantitatively, we need a technical lemma (Lemma 2.5) to estimate the closeness of line transversals to center of gravities. Intuitively, the lemma shows that if two line transversals (directed, almost parallel to each other) intersect two balls in different orders, then those oriented lines must pass very close to the center of gravity of these two balls. The proof of Lemma 2.5 is complicated, and it relies on the following simple lemma.

Lemma 2.4. Let $B$ be a unit ball in $\mathcal{R}^{d}$ with center $o=\left(o_{1}, o_{2}, \ldots, o_{d}\right)$. Let $\ell=$ $\{\ell(t) \mid t \in \mathcal{R}\}$ be the parametric equation of a line with $\ell(t)=\left(t, a t+b, c_{3}, \ldots, c_{d}\right)$. If $\ell$ intersects $B$, then $\ell\left(t_{0}\right) \in B$, where $t_{0}=\left(o_{1}+a o_{2}-a b\right) /\left(a^{2}+1\right)$, or equivalently

$$
\frac{\left(a o_{1}+b-o_{2}\right)^{2}}{1+a^{2}}+\sum_{3 \leq i \leq d}\left(c_{i}-o_{i}\right)^{2} \leq 1 .
$$

Proof. In order for line $\ell$ to intersect the unit ball $B$, it is necessary and sufficient that

$$
\min _{t \in \mathcal{R}}\left[\left(t-o_{1}\right)^{2}+\left(a t+b-o_{2}\right)^{2}+\sum_{3 \leq i \leq d}\left(c_{i}-o_{i}\right)^{2}\right] \leq 1 .
$$

We note that

$$
\begin{aligned}
f(t) & =\left(t-o_{1}\right)^{2}+\left(a t+b-o_{2}\right)^{2} \\
& =\left(a^{2}+1\right) t^{2}+2 t\left(a b-a o_{2}-o_{1}\right)+o_{1}^{2}+\left(b-o_{2}\right)^{2} \\
& =\left(a^{2}+1\right)\left(t-\frac{o_{1}+a o_{2}-a b}{a^{2}+1}\right)^{2}+\frac{\left(a o_{1}+b-o_{2}\right)^{2}}{1+a^{2}} .
\end{aligned}
$$

Thus, the minimal value of $f(t)$ is $\left(a o_{1}+b-o_{2}\right)^{2} /\left(1+a^{2}\right)$, and it is attained when $t$ equals $t_{0}=\left(o_{1}+a o_{2}-a b\right) /\left(a^{2}+1\right)$. In order for $\ell$ to intersect $B$, it is necessary and sufficient that $\ell\left(t_{0}\right) \in B$, or equivalently

$$
\frac{\left(a o_{1}+b-o_{2}\right)^{2}}{1+a^{2}}+\sum_{3 \leq i \leq d}\left(c_{i}-o_{i}\right)^{2} \leq 1 .
$$


We are ready to prove the following technical lemma, which is crucial to the proofs of Lemma 2.8 and Theorem 3.3.

Lemma 2.5. Let $P$ and $Q$ be two disjoint unit balls in $\mathcal{R}^{d}$. Assume that their center of gravity has its first coordinate equal to zero, and that the first coordinate axis $\vec{z}$ intersects both $P$ and $Q$, with $P$ first. If another line $\ell=\left\{\ell(t)=\left(t, a t+b, c_{3}, \ldots, c_{d}\right) \mid t \in \mathcal{R}\right\}$ intersects both $P$ and $Q$, with $Q$ first, then we must have $|b| \leq a$, where $a>0$.

Proof. Suppose that the centers of $P$ and $Q$ have coordinates $\left(p_{1}, p_{2}, \ldots, p_{d}\right)$ and $\left(q_{1}, q_{2}, \ldots, q_{d}\right)$, respectively. From our assumptions it follows that $p_{1}+q_{1}=0$, so we can assume $p_{1}=-\varepsilon, q_{1}=\varepsilon$, for some constant $\varepsilon$. Since the first coordinate axis $\vec{z}$ intersects $P$ before $Q$, thus $-\varepsilon<\varepsilon$, or $\varepsilon>0$.

Because $\vec{z}$ intersects both $P$ and $Q$, we have

$$
\sum_{2 \leq i \leq d} p_{i}^{2} \leq 1 \quad \text { and } \quad \sum_{2 \leq i \leq d} q_{i}^{2} \leq 1 .
$$

Because line $\ell$ intersects $Q$, by Lemma $2.4, \ell\left(t_{2}\right) \in Q$, where

$$
t_{2}=\frac{\varepsilon+a q_{2}-a b}{a^{2}+1}
$$

and furthermore

$$
\frac{\left(a \varepsilon+b-q_{2}\right)^{2}}{1+a^{2}}+\sum_{3 \leq i \leq d}\left(c_{i}-q_{i}\right)^{2} \leq 1 .
$$

Similarly, because $\ell$ intersects $P$, we have $\ell\left(t_{1}\right) \in P$, where

$$
t_{1}=\frac{-\varepsilon+a p_{2}-a b}{a^{2}+1},
$$

and

$$
\frac{\left(-a \varepsilon+b-p_{2}\right)^{2}}{1+a^{2}}+\sum_{3 \leq i \leq d}\left(c_{i}-p_{i}\right)^{2} \leq 1 .
$$

Because $\ell$ intersects $Q$ first, we have $t_{2}<t_{1}$. By inserting in the expressions for $t_{1}, t_{2}$, we get

$$
\begin{aligned}
& \frac{\varepsilon+a q_{2}-a b}{a^{2}+1}<\frac{-\varepsilon+a p_{2}-a b}{a^{2}+1} \\
& \Rightarrow 2 \varepsilon<a\left(p_{2}-q_{2}\right) \quad \Rightarrow \quad \varepsilon<\frac{a\left(p_{2}-q_{2}\right)}{2} .
\end{aligned}
$$

By adding (2) and (3) together, we get

$$
\frac{\left(a \varepsilon+b-q_{2}\right)^{2}+\left(-a \varepsilon+b-p_{2}\right)^{2}}{1+a^{2}}+\sum_{3 \leq i \leq d}\left[\left(c_{i}-q_{i}\right)^{2}+\left(c_{i}-p_{i}\right)^{2}\right] \leq 2 .
$$


Because

$$
\left(c_{i}-q_{i}\right)^{2}+\left(c_{i}-p_{i}\right)^{2} \geq \frac{\left(p_{i}-q_{i}\right)^{2}}{2}, \quad 3 \leq i \leq d,
$$

we have

$$
\begin{aligned}
\frac{\left(a \varepsilon+b-q_{2}\right)^{2}+\left(-a \varepsilon+b-p_{2}\right)^{2}}{1+a^{2}}+\sum_{3 \leq i \leq d} \frac{\left(p_{i}-q_{i}\right)^{2}}{2} & \leq 2 \\
\Leftrightarrow \quad \frac{\left(a \varepsilon+b-q_{2}\right)^{2}+\left(-a \varepsilon+b-p_{2}\right)^{2}}{2\left(1+a^{2}\right)} & \leq 1-\sum_{3 \leq i \leq d} \frac{\left(p_{i}-q_{i}\right)^{2}}{4} .
\end{aligned}
$$

Because $P, Q$ are disjoint, we get

$$
\begin{gathered}
4 \varepsilon^{2}+\sum_{2 \leq i \leq d}\left(p_{i}-q_{i}\right)^{2} \geq 4 \\
\Leftrightarrow \quad 1-\sum_{3 \leq i \leq d} \frac{\left(p_{i}-q_{i}\right)^{2}}{4} \leq \varepsilon^{2}+\frac{\left(p_{2}-q_{2}\right)^{2}}{4} .
\end{gathered}
$$

By combining (5) and (7) together, we get

$$
\begin{aligned}
\frac{\left(a \varepsilon+b-q_{2}\right)^{2}+\left(-a \varepsilon+b-p_{2}\right)^{2}}{2\left(1+a^{2}\right)} \leq \varepsilon^{2}+\frac{\left(p_{2}-q_{2}\right)^{2}}{4} \\
\Leftrightarrow b^{2}-b\left(p_{2}+q_{2}\right)+a^{2} \varepsilon^{2}+\frac{p_{2}^{2}+q_{2}^{2}}{2}+a \varepsilon\left(p_{2}-q_{2}\right) \\
\quad \leq\left(a^{2}+1\right)\left(\varepsilon^{2}+\frac{\left(p_{2}-q_{2}\right)^{2}}{4}\right) \\
\Leftrightarrow b^{2}-b\left(p_{2}+q_{2}\right)+\frac{\left(p_{2}+q_{2}\right)^{2}}{4} \leq \varepsilon^{2}-a \varepsilon\left(p_{2}-q_{2}\right)+a^{2} \frac{\left(p_{2}-q_{2}\right)^{2}}{4} \\
\Leftrightarrow\left(b-\frac{p_{2}+q_{2}}{2}\right)^{2} \leq\left(\varepsilon-\frac{a\left(p_{2}-q_{2}\right)}{2}\right)^{2} .
\end{aligned}
$$

With the help of (4), the preceding inequality immediately implies that

$$
\left|b-\frac{p_{2}+q_{2}}{2}\right| \leq \frac{a\left(p_{2}-q_{2}\right)}{2}-\varepsilon .
$$

From (1) and (6), we get

$$
\begin{aligned}
\sum_{2 \leq i \leq d}\left(p_{i}+q_{i}\right)^{2} & =2\left[\sum_{2 \leq i \leq d}\left(p_{i}^{2}+q_{i}^{2}\right)\right]-\left[\sum_{2 \leq i \leq d}\left(p_{i}-q_{i}\right)^{2}\right] . \\
& \leq 4-\left(4-4 \varepsilon^{2}\right)=4 \varepsilon^{2} .
\end{aligned}
$$

Equation (9) implies that $\left|p_{2}+q_{2}\right| \leq 2 \varepsilon$. By combining it with (8), we get

$$
|b| \leq \frac{\left|p_{2}+q_{2}\right|}{2}+\frac{a\left(p_{2}-q_{2}\right)}{2}-\varepsilon \leq \frac{a\left(p_{2}-q_{2}\right)}{2} \leq a .
$$

Since $p_{2}$ and $q_{2}$ are less than or equal to 1 , this completes the proof. 


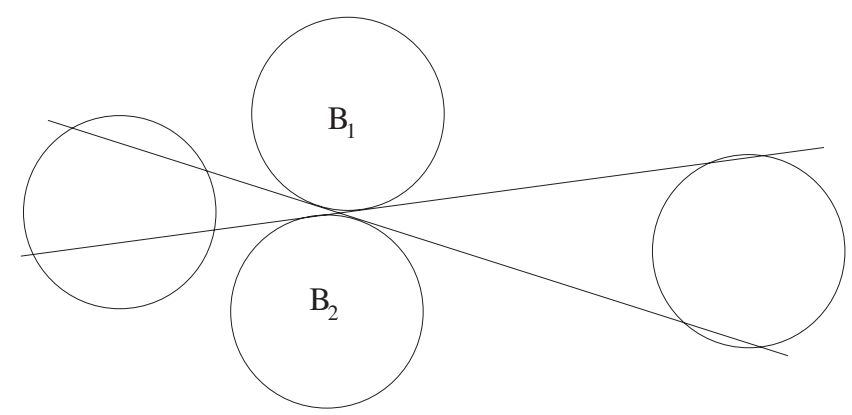

Fig. 1. An example of a switched pair $\left(B_{1}, B_{2}\right)$.

\subsection{Switched Pairs}

We say that a pair of balls $B_{1}, B_{2}$ forms a switched pair in $\mathcal{S}$ if there exist two directed line transversals $\ell$ and $\ell^{\prime}$ that meet $B_{1}, B_{2}$ in different orders. All the transversal lines are directed such that they pass through ball $a$ before ball $b$, with balls $a, b$ having the farthest distance among the family of balls. Figure 1 shows an example of a switched pair $\left(B_{1}, B_{2}\right)$. Switched pairs are obviously central to geometric permutations, and the next lemmas establish key properties that allow us to bound their number. The first lemma gives an upper bound on the distance between two balls forming a switched pair. The distance between two balls $P$ and $Q$ is defined as their pointwise minimum distance: $\operatorname{dist}(P, Q)=\min _{p \in P, q \in Q} \operatorname{dist}(p, q)$.

Lemma 2.6. The distance between two balls making a switched pair is $O\left(1 / n^{2}\right)$.

Proof. Let $\mathcal{S}$ be a set of disjoint unit balls in $\mathcal{R}^{d}$ with $\left(B_{1}, B_{2}\right)$ a switched pair of $\mathcal{S}$. Let $o_{1}, o_{2}$ be the centers of $B_{1}, B_{2}$, respectively, and let $z$ be the line passing through $o_{1}, o_{2}$. Denote $\delta=\operatorname{dist}\left(B_{1}, B_{2}\right)$ and $D=\operatorname{dist}\left(o_{1}, o_{2}\right)$. Thus, $D=2+\delta$. Because $\left(B_{1}, B_{2}\right)$ is a switched pair, there exist two directed line transversals $\ell$ and $\ell^{\prime}$ that meet $B_{1}$ and $B_{2}$ in different orders. Let $2 \varphi$ be the angle between $\ell$ and $\ell^{\prime}$. Furthermore, let $\theta$ denote the angle between $\ell$ and $z$, and $\theta^{\prime}$ denote the angle between $\ell^{\prime}$ and $z$. See Fig. 2.

By Lemma 2.2, both $\theta$ and $\theta^{\prime}$ are bounded from above by $\theta_{0}$. Here

$$
\sin \theta_{0}=\frac{2}{D}=\frac{2}{2+\delta} \quad \Longrightarrow \quad \delta=2\left(\frac{1}{\sin \theta_{0}}-1\right) .
$$

It is easy to show that $\theta+\theta^{\prime}+2 \varphi \geq \pi$. Figure 2 gives an illustration for $d=2$, where equality holds. Therefore

$$
2 \theta_{0}+2 \varphi \geq \theta+\theta^{\prime}+2 \varphi \geq \pi \quad \Longrightarrow \quad \theta_{0} \geq \frac{\pi}{2}-\varphi \quad \Longrightarrow \quad \sin \theta_{0} \geq \cos \varphi
$$

By combining (10) and (11) together, we obtain

$$
\delta=2\left(\frac{1}{\sin \theta_{0}}-1\right) \leq 2\left(\frac{1}{\cos \varphi}-1\right)=\frac{4 \sin ^{2}(\varphi / 2)}{\cos \varphi} \leq \frac{\varphi^{2}}{\cos \varphi}=O\left(\varphi^{2}\right)=O\left(1 / n^{2}\right) .
$$




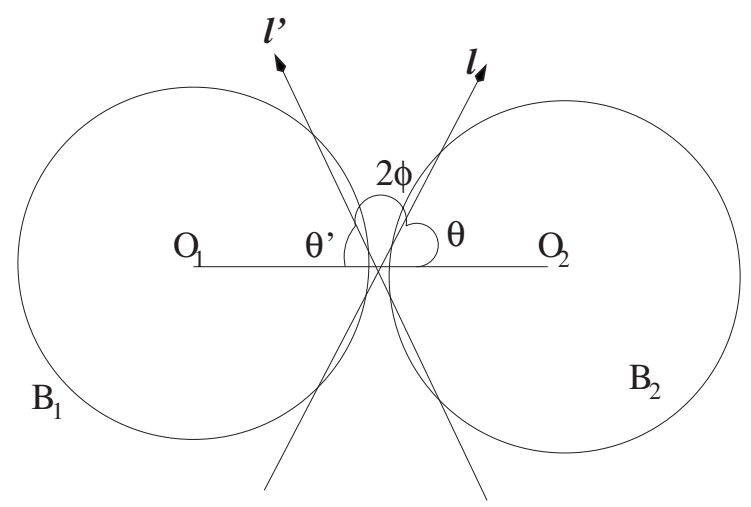

Fig. 2. The distance between $B_{1}, B_{2}$ must be very small as $\ell, \ell^{\prime}$ are almost parallel.

In the above inequalities we used the relations $1-\cos \varphi=2 \sin ^{2} \varphi / 2$ and $\sin \varphi / 2 \leq \varphi / 2$. For the last inequality, we used the fact $\varphi=O(1 / n)$, which is based on Lemma 2.3. In addition, we used the fact that $1 / \cos \varphi$ is bounded as $\varphi$ is close to 0 .

The above lemma immediately implies:

Lemma 2.7. Let $\left(B_{1}, B_{2}\right)$ be a switched pair in $\mathcal{S}$, and let $o_{1}, o_{2}$ be the centers of these balls. If $\ell$ is a line transversal of $\mathcal{S}$, then the line $\ell$ is nearly perpendicular to the line passing through $o_{1}, o_{2}$. More specifically, the angle between $\ell$ and $\overrightarrow{o_{1} O_{2}}$ is $\pi / 2-O(1 / n)$.

The following lemma establishes one of the central properties of switched pairs.

Lemma 2.8. Let $\mathcal{S}$ be a set of n pairwise disjoint unit balls in $\mathcal{R}^{d}$, where $n$ is greater than a sufficiently large constant depending on $d$. Then a ball of $\mathcal{S}$ can appear in at most one switched pair.

Proof. We prove the unique pairing argument by contradiction. Suppose that $\mathcal{S}$ is a set of disjoint unit balls in $\mathcal{R}^{d}$ with at least one directed line transversal. Let $\vec{z}$-axis denote the line connecting the centers of two farthest balls of $\mathcal{S}$. Suppose that ball $B$ forms switched pairs with at least two other balls. Specifically, assume that both $\left(B, B_{1}\right)$ and $\left(B, B_{2}\right)$ are switched pairs, where $B, B_{1}, B_{2}$ are distinct balls in $\mathcal{S}$, with centers $o, o_{1}, o_{2}$, respectively.

By Lemma 2.6, $\operatorname{dist}\left(B, B_{1}\right)=O\left(1 / n^{2}\right)$ and $\operatorname{dist}\left(B, B_{2}\right)=O\left(1 / n^{2}\right)$. Because $n$ is sufficiently large, dist $\left(o, o_{1}\right)$ and $\operatorname{dist}\left(o, o_{2}\right)$ are close to 2. By Lemma 2.7, $\overrightarrow{o o_{1}}$ and $\overrightarrow{o o_{2}}$ are almost perpendicular to the $\vec{z}$-axis. If $\pi$ is the plane passing through $o$ and perpendicular to $\vec{z}$, then both $o_{1}, o_{2}$ are very close to $\pi$. Let $g_{1}$ be the midpoint of $o o_{1}$, and let $g_{2}$ be the midpoint of $o_{2}$. Then $g_{1} g_{2}$ is parallel to $o_{1} o_{2}$ and thus very close to $\pi$. Figure 3 illustrates this in the limiting case of $n=\infty$. In addition, $\operatorname{dist}\left(g_{1}, g_{2}\right)=\operatorname{dist}\left(o_{1}, o_{2}\right) / 2 \geq 1$. By Lemma 2.5 , any line transversal $\ell$ must pass close to $g_{1}$, the center of gravity of $\left(B, B_{1}\right)$, 


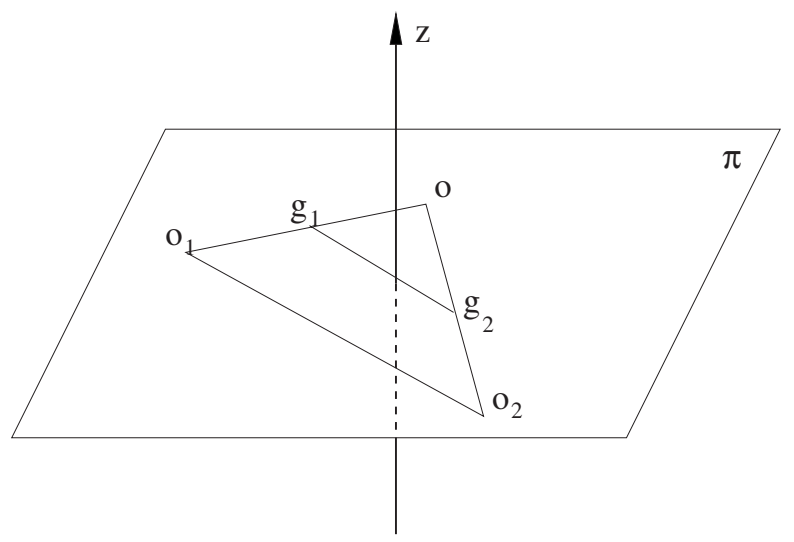

Fig. 3. Illustration of the unique pairing property with $n=\infty$.

as well as $g_{2}$, the center of gravity of $\left(B, B_{2}\right)$. Because $\ell$ is very close to both points $g_{1}$ and $g_{2}$, it is almost parallel to the line joining $g_{1} g_{2}$. Equivalently, $\ell$ is almost perpendicular to $\vec{z}$. This is a contradiction, as the angle between $\ell$ and $\vec{z}$ is bounded by $O(1 / n)$, from Lemma 2.3.

The preceding lemma shows that switched pairs are properly defined. For every ball in $\mathcal{S}$, either it belongs to a unique switched pair, or it does not belong to any switched pair. If we consider the balls in one switched pair as one object, then every oriented line transversal will meet all the objects in a fixed order. For geometric permutations, its order is fixed among all the single balls and switched pair objects. The only variation will be the order among balls inside switched pairs. Each switched pair introduces two possible orders for the oriented line transversal to meet the corresponding two balls. If there are $m$ switched pairs, then there are at most $2^{m}$ possible variations, thus at most $2^{m}$ geometric permutations. In summary, we get the following lemma.

Lemma 2.9. Let $\mathcal{S}$ be a set of $n$ disjoint convex objects in $\mathcal{R}^{d}$ where the unique pairing property holds for switched pairs. Then two members forming a switched pair must appear consecutively in all geometric permutations of $\mathcal{S}$. If there are a total of $m$ switched pairs, then $\mathcal{S}$ admits at most $2^{m}$ different geometric permutations.

\section{Geometric Permutations of Unit Balls}

In this section we establish the major result, a constant bound on geometric permutations for unit balls in $\mathcal{R}^{d}$, for $d>2$. We achieve our goal by bounding the number of switched pairs. In order to bound the number of switched pairs, we prove both a lower bound and an upper bound for the distance between different switched pairs. 
Lemma 3.1. Consider two different switched pairs: $P=\left(B_{1}, B_{2}\right)$ and $P^{\prime}=\left(B_{1}^{\prime}, B_{2}^{\prime}\right)$. Then there must exist two line transversals $\ell$ and $\ell^{\prime}$ that meet both switched pairs in different orders. In other words, there exist line transversals $\ell$ and $\ell^{\prime}$ such that $\ell$ meets $B_{1}$ before $B_{2}$ and $B_{1}^{\prime}$ before $B_{2}^{\prime}$, while $\ell^{\prime}$ meets $B_{2}$ before $B_{1}$ and $B_{2}^{\prime}$ before $B_{1}^{\prime}$, possibly after interchanging $B_{1}$ and $B_{2}$ and/or $B_{1}^{\prime}$ and $B_{2}^{\prime}$.

Proof. By definition, for the switched pair $P=\left(B_{1}, B_{2}\right)$, there exists two line transversals $\ell$ and $\ell^{\prime}$ such that $\ell$ meets $B_{1}$ before $B_{2}$ and $\ell^{\prime}$ meets $B_{2}$ before $B_{1}$. Now consider the order in which $\ell$ and $\ell^{\prime}$ meet the pair $P^{\prime}=\left(B_{1}^{\prime}, B_{2}^{\prime}\right)$. If they meet the balls of $P^{\prime}$ in different orders, then $\ell$ and $\ell^{\prime}$ are our desired line transversals. Otherwise, without loss of generality, we assume that $\ell$ and $\ell^{\prime}$ both meet $B_{1}^{\prime}$ before $B_{2}^{\prime}$.

For the switched pair $P^{\prime}=\left(B_{1}^{\prime}, B_{2}^{\prime}\right)$, there exists a line transversal $\ell^{\prime \prime}$ that meets $B_{2}^{\prime}$ before $B_{1}^{\prime}$. Now consider the order in which $\ell^{\prime \prime}$ meets the balls of $P=\left(B_{1}, B_{2}\right)$. If $\ell^{\prime \prime}$ meets $B_{1}$ before $B_{2}$, then $\ell^{\prime}$ and $\ell^{\prime \prime}$ are the desired line transversals. Otherwise, $\ell^{\prime \prime}$ meets $B_{2}$ before $B_{1}$, in which case $\ell$ and $\ell^{\prime \prime}$ are the desired line transversals. This completes the proof.

We need one more technical lemma.

Lemma 3.2. The distance between the centers of gravity of two switched pairs has a lower bound of $\sqrt{2}-\varepsilon(n)$, where $\varepsilon(n)>0$ and $\lim _{n \rightarrow \infty} \varepsilon(n)=0$.

Proof. Let $P=\left(B_{1}, B_{2}\right)$ and $P^{\prime}=\left(B_{1}^{\prime}, B_{2}^{\prime}\right)$ be two switched pairs with centers of gravity $g$ and $g^{\prime}$, respectively. Balls $B_{1}, B_{2}, B_{1}^{\prime}, B_{2}^{\prime}$ have their corresponding centers $o_{1}, o_{2}, o_{1}^{\prime}, o_{2}^{\prime}$. Because $g$ is the midpoint of segment $o_{1} o_{2}$, thus

$$
\begin{aligned}
& \operatorname{dist}\left(o_{1}^{\prime}, o_{1}\right)^{2}+\operatorname{dist}\left(o_{1}^{\prime}, o_{2}\right)^{2}=2 \operatorname{dist}\left(o_{1}^{\prime}, g\right)^{2}+\frac{\operatorname{dist}\left(o_{1}, o_{2}\right)^{2}}{2} \geq 8, \\
& \operatorname{dist}\left(o_{2}^{\prime}, o_{1}\right)^{2}+\operatorname{dist}\left(o_{2}^{\prime}, o_{2}\right)^{2}=2 \operatorname{dist}\left(o_{2}^{\prime}, g\right)^{2}+\frac{\operatorname{dist}\left(o_{1}, o_{2}\right)^{2}}{2} \geq 8 .
\end{aligned}
$$

In the above inequalities we used the fact that all the balls are pairwise disjoint. Add these two inequalities together, we get

$$
\operatorname{dist}\left(o_{1}^{\prime}, g\right)^{2}+\operatorname{dist}\left(o_{2}^{\prime}, g\right)^{2}+\frac{\operatorname{dist}\left(o_{1}, o_{2}\right)^{2}}{2} \geq 8 .
$$

Because $g^{\prime}$ is the midpoint of segment $o_{1}^{\prime} o_{2}^{\prime}$, thus

$$
\operatorname{dist}\left(o_{1}^{\prime}, g\right)^{2}+\operatorname{dist}\left(o_{2}^{\prime}, g\right)^{2}=2 \operatorname{dist}\left(g, g^{\prime}\right)^{2}+\frac{\operatorname{dist}\left(o_{1}^{\prime}, o_{2}^{\prime}\right)^{2}}{2} .
$$

By combining (12) and (13) together,

$$
\operatorname{dist}\left(g, g^{\prime}\right)^{2} \geq 4-\frac{\operatorname{dist}\left(o_{1}, o_{2}\right)^{2}+\operatorname{dist}\left(o_{1}^{\prime}, o_{2}^{\prime}\right)^{2}}{2} .
$$


From Lemma 2.6, $\operatorname{dist}\left(o_{1}, o_{2}\right)=2+O\left(1 / n^{2}\right)$ and $\operatorname{dist}\left(o_{1}^{\prime}, o_{2}^{\prime}\right)=2+O\left(1 / n^{2}\right)$. By plugging in these estimations into (14), we get

$$
\operatorname{dist}\left(g, g^{\prime}\right) \geq \sqrt{4-\left(2+O\left(1 / n^{2}\right)\right)}=\sqrt{2}-O\left(1 / n^{2}\right)=\sqrt{2}-\varepsilon(n) .
$$

This completes the proof.

Theorem 3.3. Let $\mathcal{S}$ be a set of $n$ pairwise disjoint unit balls in $\mathcal{R}^{d}$ that admits at least one line transversal. With $n$ sufficiently large, the number of switched pairs in $\mathcal{S}$ is at most 2.

Proof. Suppose that $\mathcal{S}$ admits a total number of $m$ switched pairs, with $m>1$. If we consider each switched pair (two balls) as one object, then every oriented line transversal meets these switched pairs in a fixed order. Let $P_{1}, P_{2}, \ldots, P_{m}$ denote all the switched pairs with the corresponding sorted order. Thus for any oriented line transversal, it meets $P_{1}$ first and $P_{m}$ last.

By Lemma 3.1, there exist two oriented line transversals $\ell, \ell^{\prime}$ such that the orders in which they meet the balls of $P_{1}$ and $P_{m}$ are switched. Let us choose $\ell$ as the first coordinate axis, and consider the orthogonal projection of $\ell^{\prime}$ onto a plane normal to $\ell$. Choose the direction of this projection as the second coordinate axis. We can write the equations for $\ell, \ell^{\prime}$ in the following form:

$$
\begin{array}{ll}
\ell: & \{\ell(t)=(t, 0, \ldots, 0) \mid t \in \mathcal{R}\}, \\
\ell^{\prime}: & \left\{\ell^{\prime}(t)=\left(t, a t+b, c_{3}, \ldots, c_{d}\right) \mid t \in \mathcal{R}\right\},
\end{array}
$$

where $a, b, c_{3}, \ldots, c_{d}$ are all constants.

Let $g_{i}$ denote the center of gravity for the $i$ th switched pair $P_{i}$, and $x_{g_{i}}$ is the first coordinate of $g_{i}$, for $i=1, \ldots, m$. Because line $\ell$ intersects these switched pairs in increasing order, thus $x_{g_{1}}<x_{g_{2}}<\cdots<x_{g_{m}} . \ell$ and $\ell^{\prime}$ have different transversal orders for these two balls consisting of switched pair $P_{1}$. By Lemma 2.5, $\left|a x_{g_{1}}+b\right| \leq a$. Similarly, we apply Lemma 2.5 to $P_{m}$ and get $\left|a x_{g_{m}}+b\right| \leq a$. By combining these two inequalities together, we get $a\left|x_{g_{m}}-x_{g_{1}}\right| \leq 2 a$, or $x_{g_{m}}-x_{g_{1}} \leq 2$.

By Lemma 3.2, the minimal distance between the centers of gravity of two switched pairs is at least $\sqrt{2}-\varepsilon(n)$. In the limit, when $n$ approaches infinity, all line transversals of $\mathcal{S}$ will converge to a line $z$, which contains all the centers of gravity $g_{i}$, for $i=1, \ldots, m$. Thus $x_{g_{i+1}}-x_{g_{i}}$ is an infinite approximation of the distance between $g_{i+1}$ and $g_{i}$ and $x_{g_{i+1}}-x_{g_{i}} \geq \sqrt{2}-\varepsilon(n)$ for $i=1, \ldots, m-1$. Since $m$ is the total number of switched pairs, we must have

$$
m-1 \leq \frac{2}{\sqrt{2}-\varepsilon(n)}=\sqrt{2}+\varepsilon^{\prime}(n) .
$$

Since $m$ is an integer, we conclude that $m \leq 2$ when $n$ is sufficiently large. Thus, in any dimension $d$, when $n$ is sufficiently large, the set $\mathcal{S}$ can have at most two switched pairs. 
We can now state our main theorem.

Theorem 3.4. A set $\mathcal{S}$ ofn pairwise disjoint unit balls in $\mathcal{R}^{d}$ admits at most four distinct geometric permutations, when $n$ is sufficiently large.

Proof. The theorem follows directly by combining Lemma 2.9 and Theorem 3.3.

\section{Concluding Remarks}

We resolved an open conjecture in this paper by showing that a set of $n$ unit balls in $\mathcal{R}^{d}$ admits at most four geometric permutations. This constant bound significantly improves upon the previous known bound of $O\left(n^{d-1}\right)$ for general balls.

\section{Acknowledgments}

We wish to thank Jinhui Xu for pointing out a possible improvement in our preliminary result. We thank an anonymous referee for suggesting a geometric proof to one of our lemmas.

\section{References}

1. N. Amenta, Finding a line transversal of axial objects in three dimensions, in Proceedings of the 3 rd Annual ACM-SIAM Symposium on Discrete Algorithms, pages 66-71, 1992.

2. A. Asinowski, Common transversals and geometric permutations, Master's thesis, Technion IIT, Haifa, 1998.

3. L. Danzer, B. Grunbaum and V. Klee, Helly's theorem and its relatives, Convexity, AMS, 7:101-180, 1963.

4. H. Edelsbrunner and M. Sharir, The maximum number of ways to stab $n$ convex non-intersecting sets in the plane is $2 n-2$, Discrete \& Computational Geometry, 5(1):35-42, 1990.

5. J. E. Goodman, R. Pollack and R. Wenger. Geometric transversal theory, in New Trends in Discrete and Computational Geometry, J. Pach (Ed.), vol. 10 of Algorithms and Combinatorics, pages 163-198, Springer-Verlag, Berlin, 1993.

6. M. Hohmeyer and S. Teller, Stabbing oriented convex polygons in randomized $O\left(n^{2}\right)$ time, in $C O N M$ Vol. 178: Proc. Jerusalem Combinatorics '93, pages 311-318, American Mathematical Society, Providence, RI, 1993.

7. Y. Huang, J. Xu and D. Z. Chen, Geometric permutations of high dimensional spheres, in Proceedings of the Twelfth Annual ACM-SIAM Symposium on Discrete Algorithms, pages 342-343, 2001.

8. M. Katchalski, T. Lewis and A. Liu, The different ways of stabbing disjoint convex sets, Discrete \& Computational Geometry, 7:197-206, 1992.

9. M. Katchalski, T. Lewis and J. Zaks, Geometric permutations for convex sets, Discrete Mathematics, 54:271-284, 1985.

10. M. Katz and K. Varadarajan, A tight bound on the number of geometric permutations of convex fat objects in $\mathcal{R}^{d}$, in Proceedings of the Symposium on Computational Geometry 2001, pages 249-251. Extended version appears in Discrete \& Computational Geometry, 26:543-548, 2001.

11. S. Smorodinsky, J. S. B. Mitchell and M. Sharir, Sharp bounds on geometric permutations for pairwise disjoint balls in $R^{d}$, Discrete \& Computational Geometry, 23:247-259, 2000.

12. S. Teller and C. Sequin, Visibility preprocessing for interactive walkthroughs, in Computer Graphics (Proc. Siggraph '91), 25:61-69, 1991. 
13. R. Wenger, Upper bounds on geometric permutations for convex sets, Discrete \& Computational Geometry, 5:27-33, 1990 .

14. R. Wenger, Helly-type theorems and geometric transversals, in Handbook of Discrete and Computational Geometry, J. E. Goodman and J. O'Rourke (Eds.), chapter 4, pages 63-82, CRC Press, Boca Raton, FL, 1997.

15. Y. Zhou and S. Suri, Shape sensitive geometric permutations, in Proceedings of the Twelfth Annual ACMSIAM Symposium on Discrete Algorithms, pages 234-243, 2001.

Received October 19, 2001, and in revised form January 3, 2002, and March 23, 2002. Online publication December 20, 2002. 